\title{
ESTRATEGIAS PARA LA CREACIÓN DE NUEVAS EMPRESAS, UN ENFOQUE SOCIOCULTURAL O INSTITUCIONAL
}

\author{
Enore Adriana Chávez-Jiménez ${ }^{1}$ \\ José G. Vargas-Hernández, M. B. A.; Ph.D. ${ }^{2}$
}

\begin{abstract}
RESUMEN
El presente trabajo tiene la finalidad de recapitular las principales teorías desde un enfoque sociocultural o institucional que fomentan la creación de nuevas empresas. Dado este contexto, se planteó la importancia del estudio teórico de los factores que influyen para tomar decisiones en el desarrollo de nuevas empresas y cómo afecta el entorno en el cual se desenvuelven los mismos empresarios. El método de investigación empleado es la revisión de la literatura existente mediante el análisis de las principales teorías que intervienen en la creación de empresas, enfatizándose en el enfoque sociocultural. El análisis concluye profundizando en cómo la actividad de entrepreneurship ha fomentado la creación de empresas, siendo un factor importante en el desarrollo económico del país.
\end{abstract}

PALABRAS CLAVE: EMPRENDEDOR, EMPRESA, ENFOQUE SOCIOCULTURAL, TEORÍAS SOBRE LA CREACIÓN DE EMPRESAS

\section{ABSTRACT}

This paper aims to summarize the main theories from sociocultural or institutional approach to encourage the creation of new businesses. Given this context, it raised the importance of the theoretical study of the factors that influence decision making in the development of new businesses and how that process is affected by the environment in which they operate. The research method used is to review the existing literature by analyzing the major theories involved in the creation of companies emphasizing the sociocultural approach. The analysis concludes about how the activity of entrepreneurship has encouraged the creation of companies, being an important factor in economic development.

KEYWORDS: ENTREPRENEUR, COMPANY, SOCIOCULTURAL APPROACH, THEORIES ON THE CREATION OF COMPANIES.

JEL: L26, M13.

1,2 Centro Universitario de Ciencias Económico Administrativas, Universidad de Guadalajara, Periférico Norte 799, Edif. G201-7, Núcleo Universitario Los Belenes,Zapopan, Jalisco, 45100, México,Tel. 523337703340 Ext. 25685 jvargas2006@gmail.com,jgvh0811@yahoo.com,josevargas@cucea.udg.mx 


\section{INTRODUCCIÓN}

La creación de empresas, también conocida como entrepreneurship, en la actualidad ha sido un área de un desarrollo creciente, gracias al gran aporte en el desarrollo económico que ha generado. Es por ello que surgió el interés por revisar la literatura existente dentro de este ámbito, la cual sirve de apoyo para entender la naturaleza y las características del fenómeno emprendedor, además de exponer cómo ha contribuido en el bienestar de la sociedad. A pesar de su influencia en el mejoramiento económico nacional con la apertura de nuevas empresas, algunas de estas no han sido constituidas con las bases necesarias, provocando su cierre; por ello es necesaria la ampliación del campo de la investigación referente al fenómeno del emprendedor.

El fenómeno entrepreneurship ha fortalecido el país ante la crisis económica que enfrenta así como ante la falta de oportunidades de empleo, ya que como respuesta a estas dificultades, gran parte de los ciudadanos se han enfocado en crear sus propias fuentes de ingreso mediante proyectos de autogestión, lo cual se refleja en la creación de nuevas empresas.

Las empresas, $y$ en general las organizaciones se crean con un propósito determinado como consecuencia de las oportunidades existentes. Son las instituciones, a través de las limitaciones formales e informales, junto con la estructura de derechos de propiedad de la colectividad, las que determinan las oportunidades que hay en una sociedad, $y$ por consiguiente, el tipo de empresas que se crearán. Las organizaciones y las empresas son creadas para aprovechar estas oportunidades (North, 1993:15-18).

Mediante la revisión de literatura de las principales teorías que intervienen en la creación de empresas, enfatizándose en el enfoque sociocultural o institucional, se planteó la importancia de los factores que influyen para tomar decisiones en el desarrollo de nuevas empresas y cómo afecta el entorno en donde se desenvuelven los mismos empresarios, teniendo como objetivo el presente trabajo recapitular las principales teorías desde un enfoque sociocultural o institucional que fomentan la creación de nuevas empresas.

El trabajo propone una revisión de literatura que proporcionan las diversas teorías que actualmente existen en relación con el fomento de la creación de nuevas empresas desde un enfoque de las estrategias sociocultural e institucional. En cuanto a la estructura de este trabajo, primero se habla sobre las teorías existentes en general de la creación de empresas dentro de los cuatro enfoques, describiendo brevemente cada uno, en segundo lugar se expone cada una de las teorías que conforman el enfoque sociocultural e institucional. Finalmente se abordan las conclusiones sobre la aplicación del análisis de las teorías enfocadas a la creación de nuevas empresas.

\section{TEORÍAS SOBRE LA CREACIÓN DE EMPRESAS}

La creación de nuevas empresas ha sido parte fundamental para la solución de problemas dentro del desarrollo económico del país, con la generación de empleos, innovación empresarial y el crecimiento económico mediante una ventaja competitiva. El Doctor Veciana (1999) realizó una clasificación de las principales teorías sobre el estudio de la función empresarial y la creación de empresas (entrepreneurship), en donde las agrupa según el enfoque, dando como resultado cuatro bloques: enfoque económico, enfoque psicológico, enfoque sociocultural o institucional y enfoque gerencial, estos últimos fueron clasificados en tres niveles de análisis: micro (nivel individual), meso (nivel empresas) y macro (nivel global de la economía). Veciana (1999) abarca las principales teorías y modelos para la creación de empresas. Ver figura 1.

El enfoque económico engloba las teorías que pretenden explicar la función del empresario y la creación de empresas sobre la base de la racionalidad económica (Veciana 1999).

En el enfoque psicológico se estudia al empresario como persona física creadora de una empresa, es decir, las investigaciones se orientan 
hacia lo que se ha denominado un concepto empírico del empresario (Veciana 1999).

El enfoque sociocultural o institucional se refiere a la influencia de los factores socioculturales del entorno en la creación de empresas, en este se explica cuáles son los aspectos sociales e institucionales que motivan hacia la actividad emprendedora. Así, esta propensión puede venir explicada por determinados fenómenos sociales como la marginalidad social, la religión, la familia, la ideología reinante, el entorno empresarial, la cultura, etc. (Santos, 1998). Las diversas teorías de este enfoque concluyen que si la función empresarial ha florecido, debe haber una congruencia entre la construcción ideológica-cultural y el comportamiento económico (Amit, et al., 1993).

FIGURA 1

ENFOQUES TEÓRICOS SOBRE EL ESTUDIO DE LA FUNCIÓN EMPRESARIAL Y LA CREACIÓN DE EMPRESAS ("ENTREPRENEURSHIP")

\begin{tabular}{|c|c|c|c|c|}
\hline $\begin{array}{l}\text { Enfoques } \\
\text { Nivel de Análisis }\end{array}$ & $\begin{array}{l}\text { ENFOQUE } \\
\text { ECONÓMICO }\end{array}$ & $\begin{array}{l}\text { ENFOQUE } \\
\text { PSICOLÓGICO }\end{array}$ & $\begin{array}{l}\text { ENFOQUE } \\
\text { SOCIOCULTURAE } \\
\text { INSTITUCIONAL }\end{array}$ & $\begin{array}{l}\text { ENFOQUE } \\
\text { GERENCIAL }\end{array}$ \\
\hline $\begin{array}{l}\text { MICRO (Nivel } \\
\text { individual) }\end{array}$ & $\begin{array}{l}\text { La función } \\
\text { empresarial como } \\
\text { cuarto factor de } \\
\text { producción } \\
\text { Teoría del beneficio } \\
\text { del empresario }\end{array}$ & $\begin{array}{l}\text { Teoría de los rasgos } \\
\text { de la personalidad } \\
\text { Teoría } \\
\text { psicodinámica de } \\
\text { la personalidad del } \\
\text { empresario }\end{array}$ & $\begin{array}{l}\text { Teoría de la marginación } \\
\text { Teoría del rol } \\
\text { Teoría de redes }\end{array}$ & $\begin{array}{l}\text { Teoría de la eficiencia } \\
\text { X de Liebenstein } \\
\text { Teoría del } \\
\text { comportamiento del } \\
\text { empresario } \\
\text { Modelos de proceso } \\
\text { de la creación de } \\
\text { empresas }\end{array}$ \\
\hline $\begin{array}{l}\text { MESO (Nivel de } \\
\text { empresas) }\end{array}$ & $\begin{array}{l}\text { Teoría de costos de } \\
\text { transacción }\end{array}$ & & $\begin{array}{l}\text { Teoría de redes } \\
\text { Teoría de la incubadora } \\
\text { Teoría evolucionista }\end{array}$ & $\begin{array}{l}\text { Modelos de éxito de la } \\
\text { nueva empresa } \\
\text { Modelos de generación } \\
\text { y desarrollo de nuevos } \\
\text { proyectos innovadores }\end{array}$ \\
\hline $\begin{array}{l}\text { MACRO (Nivel global } \\
\text { de la economía) }\end{array}$ & $\begin{array}{l}\text { Teoría del } \\
\text { desarrollo } \\
\text { económico de } \\
\text { Schumpeter }\end{array}$ & $\begin{array}{l}\text { Teoría del } \\
\text { empresario de } \\
\text { Kirzner }\end{array}$ & $\begin{array}{l}\text { Teoría del desarrollo } \\
\text { económico de Weber } \\
\text { Teoría del cambio social } \\
\text { Teoría de la ecología } \\
\text { Teoría institucional }\end{array}$ & \\
\hline
\end{tabular}

Fuente: Veciana, J.M. (1999:16)

En el enfoque gerencial se engloban las teorías relacionadas con la labor de los empresarios en el proceso de la creación de nuevas empresas. Dentro de este enfoque se encuentran las teorías enfocadas a la aplicación de los conocimientos por parte de los empresarios, enmarcando los modelos útiles para la creación de nuevas empresas.

\section{TEORÍAS CON UN ENFOQUE SOCIO- CULTURAL O INSTITUCIONAL}

Como ya se mencionó anteriormente, en este enfoque se engloban las teorías que explican cuáles son los aspectos sociales e institucionales que motivan la actividad emprendedora. Debido a su gran extensión nos enfocaremos 
en las teorías insertas en este enfoque, entre las cuales se encuentran: la teoría de la marginación, la teoría del rol, la teoría de redes, la teoría de la incubadora, la teoría de la ecología y la teoría institucional.

\subsection{Teoría de la marginación}

Esta teoría considera la importancia de los factores externos, principalmente los negativos, a los cuales se podría enfrentar el empresario. La teoría se origina en una serie de investigaciones donde saltaron a la luz sucesos contraproducentes que permitieron el desarrollo de nuevas empresas. Se encontró que los individuos más propensos a crear su propia empresa, a partir de esos factores negativos, son marginados o inadaptados, como consecuencia de un cambio en su estilo de vida anterior. Dicho fenómeno se denomina también deterioro del rol (Collins et al., 1964) o suceso disparador (Shapero, 1982).

Según esta teoría para convertirse en empresario son necesarias dos condiciones (Brunet y Alarcón, 2004):

- Un periodo de incubación, de maduración de la idea desde hace algún tiempo.

- Un suceso disparador que desencadene el proceso de creación de una empresa, un factor considerado negativo. Este tipo de eventos podría ser el desempleo de larga duración, el despido, falta de seguridad en el empleo, situaciones de rechazo de ideas, etc. (Tervo, 2006).

\subsection{Teoría del rol}

La teoría del rol trata de explicar cómo en algunas zonas geográficas existe mayor actividad de emprendurismo, mientras que en otras zonas es escasa. El entorno en el que predomina la abundancia de un sector industrial o bien la existencia de modelos emprendedores a seguir produce un efecto de arrastre que estimula la aparición de más empresarios (Nueno, 1996).

Existe una serie de factores del entorno que contribuyen a la creación de nuevas empresas (Veciana, 1999):
- Hechos que fomenten una cultura empresarial.

- Existencia en el mercado.

- Actitud favorable de la sociedad hacia la figura del empresario. Valoración.

- Disponibilidad de recursos.

- Disponibilidad de mano de obra cualificada $y$ directivos.

- Acceso a la financiación externa y capital riesgo.

- Acceso a centros de formación y servicios de asesoramiento.

- Recompensas a la función empresarial: económicas y sociales.

- Factores institucionales: actitud favorable y medios.

- Políticas de fomento.

\subsection{Teoría de redes}

La teoría de redes implica que para el desarrollo de nuevas empresas es necesario establecer una relación entre los emprendedores con las redes sociales, con el objetivo de interactuar y cooperar con otros para la obtención de los recursos imprescindibles. Una red es un sistema coordinado de relaciones de intercambio establecido por diferentes empresas especializadas en las distintas actividades de la cadena de valor del producto (Fernández y Junquera, 2001).

La teoría de redes plantea que el conjunto de relaciones específicas entre varios grupos o actores proporciona múltiples interconexiones $y$ reacciones en cadena, cuyo resultado es hacer circular la información y las ideas, $y$ facilitar al emprendedor la creación de la empresa. Las relaciones que constituyen las redes son las que existen entre el empresario, el cliente, los proveedores, entidades de crédito, organismos públicos, amigos, familiares, instituciones empresariales, etc. (Veciana, 1988) estudia a las redes en cinco dimensiones: tamaño, densidad, grado de alcance, heterogeneidad y centralidad de los nodos.

Las redes se clasifican de diversas maneras, según distintos autores. Birley (1985) distingue entre redes formales (bancos, profesionales, cámaras de comercio) e informales 
(familias, amigos, compañeros de trabajo), manifestando que los nuevos empresarios se sirven más de estas últimas que de las redes formales. Szarka (1990) diferencia entre: redes de intercambio (constituidas por empresas y organizaciones con las cuales el empresario tiene relaciones comerciales); redes de comunicación (constituidas por los individuos y las organizaciones con las que el empresario no mantiene vínculos comerciales, pero le informan de aspectos del negocio); $y$ redes sociales (constituidas por familia $y$ amigos).

Curran et al. (1993) distingue entre redes obligatorias (aquellas a las que debe pertenecer el empresario para poder sobrevivir) y voluntarias (si ser obligatorias para su supervivencia, refuerzan su posición en el mercado). Asimismo, Bryson et al. (1993) las clasifica en: redes de demanda (clientes, nuevos negocios $y$ contactos); redes relacionadas con la oferta $y$ la cooperación con otras empresas suministradoras; $y$ redes concernientes con el apoyo que suministran amigos y familia.

\subsection{Teoría de la incubadora}

La necesidad de crear y de desarrollar empresas ha animado a los gobiernos locales $y$ regionales, universidades, cámaras de comercio, empresas privadas e incluso a organizaciones no gubernamentales, al establecimiento $y$ desarrollo de programas de incubadoras (OCDE, 1999).

La teoría de la incubadora destaca la importancia que poseen las organizaciones incubadoras como elementos de estímulo empresarial. De acuerdo con esta teoría, la puesta en marcha de iniciativas empresariales viene determinada por la existencia de ciertas organizaciones que actúan como incubadoras de una idea de negocio durante un periodo de tiempo, hasta que, una vez madurada $y$ demostrada su viabilidad, se materializa en una nueva empresa.

La National Business Incubation Association (NBIA) es la organización gremial más grande que se dedica a la incubación de empresas y al fomento de la capacidad emprendedora. Según NBIA, una incubadora de empresas es una herramienta del desarrollo económico, diseñada para acelerar el nacimiento y el crecimiento de emprendimientos a través del aporte de recursos y servicios que sirven de soporte para la empresa incipiente. Provee del acceso a espacio con renta apropiada y alquileres flexibles, servicios de oficina $y$ servicios de soporte de equipos tecnológicos y asistencia para obtener el financiamiento necesario para el crecimiento de la empresa.

El Doctor José María Veciana Verges de la Universidad de Barcelona, quien hace una muy interesante reflexión sobre los temas de creación de empresas, afirma que según esta la teoría, la existencia de determinadas organizaciones (empresas industriales, centros de investigación o universidades) determinaría no solo el número de nuevas empresas en una determinada zona, sino la naturaleza de las mismas. El objetivo fundamental en las incubadoras es concebir empresas sanas hacia el desarrollo económico de la región, mediante la capacitación, consulta, investigación e infraestructura, para la generación de conocimientos, desarrollando una ventaja competitiva sostenible.

\subsection{Teoría de la ecología de la población}

La teoría de la ecología de la población o de la ecología de las organizaciones trata de explicar cómo influye el entorno que determina el ciclo de vida de las empresas; nacimiento, crecimiento y muerte. Esta teoría planteada por autores como Hannan y Freeman (1977) y reafirmada por Baumol (1993) considera que el éxito en la creación de empresas está sobre todo determinado por el entorno más que por la propia habilidad o decisión de ser empresario.

Veciana (1988) menciona entre los supuestos básicos de esta teoría que las formas de organización existentes en un momento determinado son incapaces de adaptarse a los cambios del entorno; además las modificaciones del entorno generan nuevas empresas; del mismo modo, las variaciones en las poblaciones de empresas obedecen a procesos demográficos de creación y disolución de estas.

Bygrave (1993) expone que la ecología de la población proporciona un modelo para 
la función empresarial tratando de predecir la probabilidad de nacimientos y muertes dentro de una población de empresas.

\subsection{Teoría económica institucional}

La teoría institucional trata de explicar cuáles son los aspectos sociales e institucionales que determinan a los individuos a emprender. Mientras las instituciones proveen el apropiado campo para que el crecimiento económico tenga lugar, el emprendedor será el mecanismo que genere este crecimiento (Boettke y Coyne, 2006). La existencia de una buena estructura institucional en un país ha permitido una mayor creación de empresas y un crecimiento económico superior respecto a otros países de similares condiciones, pero carentes de dicha estructura (Sobel, 2008). La adopción de instituciones tiene que preceder al comportamiento productivo del emprendedor, pues las instituciones son las que permiten la correcta instauración de empresas (Baumol, 2002).

Los autores Veblen (1904) o Commons (1934) dan origen a la teoría institucional a principios del siglo pasado, al observar la importancia económica de las leyes, hábitos o costumbres que conformaban el marco institucional en el donde se producían los hechos económicos. Pero es hasta 1990 con North, cuando revive la importancia del institucionalismo, para explicar el cambio y comportamiento de las organizaciones en función de las normas establecidas por las instituciones, pudiéndose convertir estas últimas en variables que expliquen el grado de desarrollo empresarial en los países. Diferencias en las instituciones y en las políticas de intervención, implican diferencias en el desarrollo empresarial de los países (Acemoglu y Robinson, 2000).

La teoría económica institucional de North de 1990 a 2005 explica los cambios económicos e institucionales que surgen en un determinando entorno. Principalmente, analiza el desempeño económico en función de la evolución de las instituciones y de la interacción entre las organizaciones políticas, económicas, sociales y educativas (North 1993:3). Esta teoría considera las instituciones como los incentivos para crear una estructura económica productiva que permita el fortalecimiento de las economías. Para North (1990:3) son "las instituciones las que determinan las reglas de juego de una sociedad". Peng (2010:93) construye sobre la metáfora de "las reglas del juego". Douglass North (1993), ganador del Premio Novel de economía, define mas formalmente las instituciones como "las restricciones humanamente planeadas que estructuran la interacción humana".

En cuanto al estudio de la creación de empresas desde la perspectiva de la teoría económica institucional, algunos autores distinguen dos tipos de factores: los formales $y$ los informales, ver figura 2 .

FIGURA 2

DIMENSIONES DE LAS INSTITUCIONES

\begin{tabular}{lll}
\hline Grado de formalidad & Ejemplos & Pilares de soporte \\
\hline Instituciones formales & $\begin{array}{l}\text { Leyes } \\
\text { Reglamentos } \\
\text { Reglas }\end{array}$ & Reguladores (coercitivos) \\
\hline & $\begin{array}{l}\text { Normas } \\
\text { Culturas }\end{array}$ & $\begin{array}{l}\text { Normativos } \\
\text { Cognitivos }\end{array}$ \\
\hline Enstituciones informales & Ética & \\
\hline
\end{tabular}

Fuente: Peng (2010:93) 


\section{CONCLUSIÓN}

En el análisis de literatura llevado a cabo a lo largo del artículo se ha visto como la actividad de entrepreneurship ha fomentado en los individuos la creación de nuevas empresas, siendo estas un factor importante para el desarrollo económico del país, mediante la generación de empleos y estimulando la innovación empresarial.

En este trabajo se ha podido observar como el conjunto de teorías, desde un enfoque sociocultural o institucional, pueden construir un marco teórico de referencia válido para el estudio de los factores que influyen en la creación de nuevas empresas.

Entre las teorías más influyentes dentro de este ámbito se encuentran: la teoría de la marginación, en donde se considera a los sucesos negativos que permiten el desarrollo de nuevas empresas; la teoría del rol, esta explica los factores en una zona geográfica determinada que fomentan la actividad empresarial; la teoría de redes, la cual implica la necesidad de impulsar las relaciones con el objetivo de interactuar y cooperar con las empresas para la obtención de los recursos necesarios; la teoría de la incubadora, misma que enfatiza la relevancia de las organizaciones incubadoras como el gobierno, universidades $y$ hasta empresas no gubernamentales en el estímulo empresarial, permitiendo la creación de nuevas empresas; la teoría de la ecología de la población, la cual explica cómo afecta el entorno en el ciclo de vida de las empresas: nacimiento, crecimiento $y$ muerte; $y$ por último la teoría económica institucional, esta realiza mas énfasis en la creación de nuevas empresas, con la importancia del institucionalismo, explica el cambio y comportamiento de las organizaciones en función de las normas establecidas por las instituciones, analiza el desempeño económico en función de la evolución de las instituciones y de la interacción entre las organizaciones.

El enfoque sociocultural o institucional proporciona la influencia de los factores socioculturales del entorno en la creación de empresas, explica los aspectos sociales e institucionales que motivan la actividad emprendedora.
Las diversas teorías de este enfoque concluyen que si la función empresarial ha florecido, debe haber una congruencia entre la construcción ideológica-cultural y el comportamiento económico.

\section{REFERENCIA BIBLIOGRÁFICA}

Acemoglu, J.S. Y Robinson, J.A. (2000). The colonial origins of comparative development: an empirical investigation. MIT Manusonto.

Amit, R.; Glosten, L. Y Muller, E. (1993): "Challenges to Theory Development in Entrepreneurship Research". Journal of Management Studies, Vol. 30, No. 5, pp. 815-834, Basil Blackwell Ltd.

Baumol, W.J. (1993). Entrepreneurship: productive, unproductive and destructive. Entrepreneurship, Management, and the Structure of Payoffs. Cambridge, Mass.: MIT Pres.

Baumol, W.J. (2002). The Freemarket Innovation Machine: Analyzing the Growth Miracle of Capitalism. Princeton University Press, Princeton, NJ.

Birley, S. (1985): The role of Networks in the entrepreneurial process, Journal of Business Venturing, vol. 1 (pp. 107-117).

Boettke, P.J. Y Coyne, C., (2006). Entrepreneurial behavior and institutions, en Minniti, M. (Ed.), Entrepreneurship: The Engine of Growth Perspective Series, vol. 1. Greenwood Publishing.

Brunet, I. Y Alarcón, A. (2004). Teorías sobre la figura del emprendedor, Papers 73 (pp. 81-103).

Bryson, J; Wood, P. Y Keeble, D (1993). Business networks, small firm flexibility and regional development in UK business services, Entrepreneurship and Regional Development, vol. 5 (pp. 265-77).

Bygrave, W. (1993). Theory building in the entrepreneurship paradigm, Journal of Business Venturing, no 8 (pp. 255-280).

Collins, O.F.; Moore, D.G. Y Unwala, D.B. (1964). The organization makers: a behavioural study of independent entrepreneurs. New York: Meredith. 
Commons, J.R. (1959). Institutional Economics (1934), 2 vols., Madison (Wisconsin), The University of Wisconsin Press.

Curran, J.; Jarvis, R.; Blackburn, R. Y Black, S. (1993): Networks and small firms: constructs, methodological strategies and some findings, International Small Business Journal, 11 (2) (pp. 13-25)

Fernández, E. Y Junquera, B. (2001). "Factores determinantes en la creación de empresas: una revisión de la literatura", Papers de Economía Española, no 89-90 (pp. 322-342).

Hannan, M. Y Freeman, J.H. (1977). The population ecology of organizations, American Journal of Sociology, vol. 82, no 5 (pp. 86-118).

North, D.C. (1990): Institutions, Institutional Change, and Economic Performance. Cambridge: Cambridge University Press.

North, D.C. (1993): Economic Performance through Time. Prize Lecture to the memory of Alfred Nobel, Abril 2002. Obtenido Mayo 13, 2012, de http:// members.shaw.ca/compilerpress $1 /$ A n n o \% $20 \mathrm{North} \% 20 \mathrm{Ec}$ on $\% 20$ Perform\%20thru\%20Time.htm\#Douglas

North, D. C. (1993): Instituciones, Cambio Institucional y Desempeño Económico, México, Fondo de Cultura Económica.

Nueno, P. (1996). Evolución de los conceptos de management, Management Review, no 1, enero-abril 1996 (pp. 73- 83).

Ocde (1999). Estimular el espíritu empresarial, OCDE, París.
Peng, M. (2010). Estrategia global. Segunda edición. México: Cengage Learning Editores, S.A. de C.V.

Santos Cumplido, F. J. (1998): La Teoría de la Función Empresarial: Una aproximación cualitativa al empresario sevillano. Tesis Doctoral . Universidad de Sevilla.

Shapero, A. (1982). Social Dimensions of Entrepreneurship en C. Kent et al. (eds.), The Encyclopedia of Entrepreneurship, (pp. 72-90), Englewood Cliffs, NJ: Prentice Hall.

Sobel, R.S. (2008). Testing Baumol: Institutional quality and the productivity of entrepreneurship, Journal of Business Venturing, Vol. 23 (6) (pp. 641-655).

Szarka, J. (1990). Networking and small firms, International Small Business Journal, no 2 (pp. 10-22).

Tervo, H. (2006). Regional unemployment, selfemployment and family background, Applied Economics 38 (9) (pp. 10551062).

Veblen, T. (1904). The theory of business enterprise, New York.

Veciana, J.M. (1988). "Empresario y proceso de creación de empresas", Revista Económica de Cataluña, num.8, mayoagosto (pp. 2-34).

Veciana, J.M. (1999): “Creación de Empresas como programa de investigación Científica". Revista Europea de Dirección y Economía de la Empresa, Vol. 8, No. 3, pp. 11-36. 